\title{
The role of occlusion in reducing multistability in moving point-light displays
}

\author{
DENNIS R. PROFFITT, BENNETT I. BERTENTHAL, and RALPH J. ROBERTS, JR. \\ University of Virginia, Charlottesville, Virginia
}

\begin{abstract}
Two experiments examined the role of occlusion in reducing perceived multistability in computergenerated point-light walker displays. In Experiment 1, it was found that point-light walker displays lacking occlusion were perceived as multistable and that the addition of occlusion served to reduce this perceived ambiguity. In Experiment 2, it was found that occlusion served two functions in the perception of point-light walker displays: specifying depth order and indicating the presence of implicit occluding forms. These results are discussed in relation to contemporary motion information processing models. All existing models rely solely on the motion and topographical information present in our nonoccluding display; in so doing, these models prove inadequate, since they seek to derive unique perceptions from information seen by observers as being multistable. The findings from the present experiments demonstrate that observers utilize occlusion to reduce multistability, and, thus, rules for processing this information, and/or other forms of constraining information, need to be added to existing motion information processing models.
\end{abstract}

From a mathematical point of view, any twodimensional scene is indefinitively ambiguous when construed as a projection of a three-dimensional object. Yet, except for the well-known set of ambiguous figures (Necker cube, Schroder stairs, etc.), most twodimensional images are typically seen as having unique interpretations. Static scenes are disambiguated through the implementation of such interpretative constraints as pictorial depth cues, familiarity with the objects depicted, and knowledge of representational conventions. An analogous situation is found with moving scenes. One of the most striking findings is the kinetic depth effect (KDE), in which observers accurately perceive threedimensional rigid objects when viewing transforming twodimensional distal patterns, even without the use of any of the aforementioned static picture perception cues (Wallach \& O'Connell, 1953/1976). Equally noteworthy is Johansson's (1973) finding that observers can identify the human form when they view a two-dimensional array of point-lights attached to the major joints and head of an unseen walking person.

Demonstrating that observers can extract threedimensional structure when they view moving twodimensional patterns does not provide an explanation of how they achieve unique interpretations. Strictly speaking, the stimulus information available in moving stimulus arrays typically used in motion perception research is ambiguous. Interpretative constraints are required in order to precisely delimit the set of reported percepts from

This research was supported by NICHD Grant HD-16195. We thank Karen Smith and Nancy Spetner for their assistance in running the experiments. Myron Braunstein, James Cutting, and Donald Hoffman provided valuable criticism on an earlier version of this paper. Reprint requests should be addressed to Dennis Proffitt, Department of Psychology, Gilmer Hall, University of Virginia, Charlottesville, VA 22901. the far larger set of alternatives that are, in principal, possible but not reported by observers of these moving displays.

The focus of the present paper is on examining the interpretative constraints required to extract connectivity and three-dimensional form from moving point-light displays. In particular, we investigated the role of occlusion in constraining the number of interpretations seen in moving arrays of point-lights mimicking human gait.

\section{Interpretative Constraints on Motion Information Processing}

Interpretative constraints can be thought of in two ways. First, they may represent only the perceptual system's sensitivity to constraints that are manifest in the environment. That is, from a direct perception point of view, interpretative constraints mirror environmental constraints. A second view on interpretative constraints places them within the perceptual system, making them a part of its information processing heuristics. At present, we can find no good empirical evidence to support one of these perspectives over the other. Two types of interpretative constraints on motion information processing have been proposed. First, constraints can function to select interpretations, from a set of derived possibilities, based upon some evaluative criterion such as a minimum principal. Restle's (1979) adaptation of coding theory for moving point-light displays provides such an evaluative constraint. The second type of restriction consists of processing constraints that eliminate alternatives by making heuristic assumptions that are operative during the information extraction process. Uliman's (1979) rigidity assumption is an example of such a processing constraint. Ullman showed that, if four noncoplanar points are assumed to be rigidly connected, then three distinct views of them, in parallel projection, are sufficient to define a unique 
three-dimensional interpretation of their figural relations. (This interpretation excludes considerations of reflection in depth and of size.) Although this model works for the $\mathrm{KDE}$, in which rigid objects are rotated, it fails to be sufficiently general to account for perceptions of nonrigid objects. In particular, Ullman's model cannot derive interpretations for moving jointed objects, such as a walking person.

\section{Interpretative Constraints for Extracting Structure from Point-Light Walker Displays}

Johansson (1973) introduced point-light walker displays in which the distal patterns consisted of 10-12 point-lights attached to the major joints of an unseen walking person. He found that these displays provided observers with compelling impressions of locomoting humans. In a later investigation, Johansson (1976) found that 0.1-0.2 sec was a sufficient exposure duration for perceiving the human body. Cutting and Kozlowski (1977) showed that friends could be recognized by their gait in point-light walker displays, and Cutting, Proffitt, and Kozlowski (1978) reported evidence of gender recognition in these reduced stimuli. The tractability of point-light walker displays has been greatly facilitated by the development of methods for their generation through computer synthesis (Bertenthal \& Kramer, 1984; Cutting, 1978).

From a mathematical standpoint, point-light walker displays are ambiguous and do not represent any unique three-dimensional object. Johansson (1973, 1976), however, found no evidence of multistability. Multistability refers to the perception of more than one stable interpretation in a single distal stimulus (Attneave, 1971). In seeking constraints on perceptual processing that would reduce the infinite number of possible interpretations to the single percept of appropriate biomechanical structure, Johansson proposed a perceptual vector analysis model that parsed absolute motions into relative and common motion components. Johansson's model analyzes manifest absolute motions into a common vector present in all point-lights and a set of relative motion vectors that describe the twisting and pendular movements of the body's component structures. The common motion vector specifies a perceived observer-relative displacement and serves as a frame of reference for the set of relative motions providing information for object recognition. As a processing model, this formulation is suggestive at best, because it fails to specify the connectivity between appropriate point-lights and, moreover, fails to derive any three-dimensional interpretation.

Proffitt and Cutting (1980, Cutting \& Proffitt, 1981) elaborated on Johansson's $(1973,1976)$ model. They proposed that the perceptual system performed a logical sequence of information extraction, beginning with relative motions as they occurred about centers of moment. A further constraint to this model was a minimum principal applied to relative and common motion components (Cutting \& Proffitt, 1982). As with Johansson's approach, Proffitt and Cutting's model does not specify constraints that determine which point-lights should be related. Thus, the model assumes, for example, that the motions of the hip and knee point-lights are related; however, there is nothing in the model to eliminate the relating of the wrist and knee motions. Proffitt and Cutting's approach also fails to derive any three-dimensional interpretations.

Computer vision researchers have proposed more adequate processing models for displays of moving jointed objects, and their models take two quite different approaches. One approach, which might be called a topdown model, takes the human form as given and seeks to match the presented figure to the known model (O'Rourke \& Badler, 1980). This model lacks generality, however, because it cannot recognize any pattern other than that of walkers. The second approach operates in a more bottom-up fashion and seeks to discover the connectivity in the presented pattern by employing a set of assumptions about the motion of rigidly related points rotating in different planes of depth (Hoffman \& Flinchbaugh, 1982; Webb \& Aggarwal, 1982). (These models are formally equivalent, although Webb and Aggarwal's approach is the more general of the two.) These models utilize a fixed axis assumption as their basic processing constraint (Hoffman \& Flinchbaugh call this constraint the planarity assumption). In essence, the models seek to recover rigid relations between pairs of points by testing whether or not each pair is rigidly connected and is translating or rotating about a fixed axis in some plane of depth. Whenever pairs of points are found to meet this assumption, they are interpreted as being rigidly connected. Whenever the motions of two points fail to fit the fixed axis assumption, the points are interpreted as not being connected. After deriving a set of pairwise connections, these models proceed to connect pairs having one point in common. Consider, for example, how these models find connectivity for a point-light walker. In examining all possible pairings of the point-lights, these models will find that a knee has pairwise rigid structure with an ankle and also with a hip point-light. The hip will be found to be connected with the shoulder, the shoulder with the elbow, and so forth. Thus, the fixed axis assumption represents an extremely general processing constraint enabling the extraction of coherent, three-dimensional structure from projections of rigid or jointed moving objects.

Two sources of ambiguity still remain, even after the application of the fixed axis constraint. The first is illustrated in Figure 1. Figure 1A shows an image of a pointlight walker, and Figures 1B, 1C, and 1D represent discrete images of four points within the walker display, selected at $90^{\circ}$ intervals during the gait cycle. Figure 1B depicts four point-lights moving as if attached to the shoulders and elbows of the person shown in Figure 1A, who appears to be walking on a treadmill. Point-lights $A$ and $X$, the shoulders, oscillate back and forth, $180^{\circ}$ out of phase. Point-light $B$ swings with a pendular motion about $\mathrm{A}$, and point-light $\mathrm{Y}$ moves in a similar manner about $\mathrm{X}$. These latter point-lights are the elbows. Figure $1 \mathrm{C}$ shows 
A

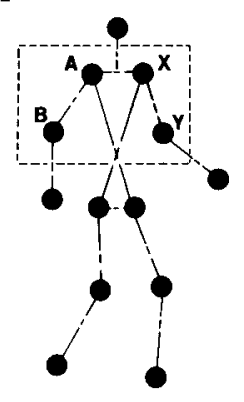

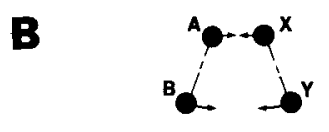

C
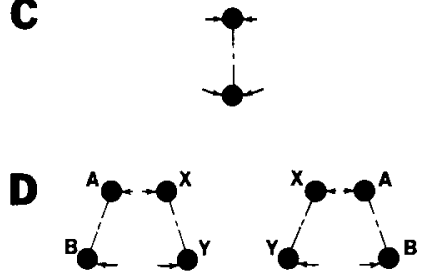

Figure 1. Panel A depicts point-lights moving as if attached to a person walking on a treadmill. The point-lights are not occluded by the form of the walker, but rather appear continuously. Points $A$ and $B$ are attached to the upper arm on one side of the body and points $X$ and $Y$ are similarly attached to the same limb on the other side. In panels B, C, and D, the shoulder and elbow lights, enclosed with the dotted lines of panel $A$, are shown in three phases of the gait cycle, separated by $90^{\circ}$. Panel B represents the positions of the two pairs of points at the moment of their greatest separation. Panel $\mathbf{C}$ shows the points at the moment when the two shoulder and elbow lights coincide. This overlapping of one point-light by another leads to more than one interpretation as the lights separate. To the left in panel $D$, is shown point-lights $A B$ and $X Y$ recoiling after contact. To the right is shown the biomechanical interpretation in which the points pass through each other.

the alignment, occurring twice a cycle, in which $\mathrm{AB}$ and $X Y$ coincide. This overlapping of one element by another leads to more than one interpretation as the elements begin again to separate. Figure 1D depicts two of the most likely interpretations of the event. To the left is shown an interpretation in which point-lights $\mathrm{AB}$ recoil after contact with $\mathrm{XY}$, and the latter two point-lights likewise reverse their velocities. The drawing to the right depicts the biomechanical interpretation in which the points pass through each other, presumably in different planes. Aggarwal and Martin (1981, Figure 6.2a-d) provide a similar analysis for pairwise-rigid structures on spinning disks. The fixed axis assumption cannot distinguish reversals in direction from continuous velocities where pairwise-rigid structures coincide.

A second source of ambiguity, which has been acknowledged by the proponents of both of the previously described models, is illustrated in Figure 2. Even when an interpretation of the previously described event is selected, one in which the two rigid pairs are separated in depth and pass through each other, the order of their depth relations remains ambiguous. That is, the fixed axis assumption derives interpretations that exclude considerations of depth order. Figure 2A depicts two possibilities: The plane containing point-lights $A B$ maintains a constant depth order, either in front of or behind the plane bearing XY. Figure 2B shows a second set of interpretations: The rigid pairs, $\mathrm{AB}$ and $\mathrm{XY}$, may maintain a constant depth relation while their depth order is continuously changing. In this case, the pairs would be seen as revolv- ing in depth, $180^{\circ}$ out of phase. Note that direction of rotation is ambiguous (Braunstein, 1983).

The two problems discussed above are not obviated by the addition of point-lights to the other joints of the unseen person in the display. At present there are no processing models sufficiently powerful to disambiguate pointlight walker displays.

This state of affairs suggests that we should seek to define additional constraints in the distal displays that may further constrain their ambiguity and assess observer sensitivity to this added information. In considering this imperative, we find that typical point-light walker displays contain occlusion information, a powerful constraint not utilized in existing processing models of this phenomenon.

\section{Occlusion}

Since Johansson's $(1973,1976)$ displays were fabricated by attaching luminance sources to real people, many of these point-lights were successively covered and uncovered from sight by the walkers' bodies. When, for example, a point-light walker translates along a path normal to an observer's line of sight, the far shoulder and hip point-lights are concealed entirely and the far elbow, wrist, knee, and ankle point-lights appear and disappear as they are occluded and disoccluded by the body.

Occlusion provides a means for referencing point-lights with regard to depth order. There is considerable evidence from studies of other phenomena that people can make appropriate use of such information. White and Mueser (1960) employed a shadow caster technique (Metzger, 1934) to present rotating configurations of elements in their studies on the kinetic depth effect. They found that when the elements were identical, the stimuli were ex-

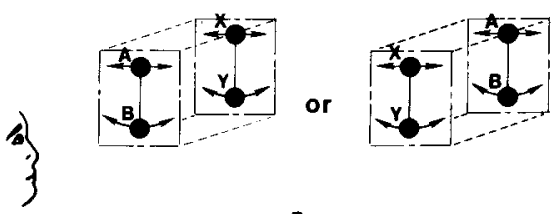

A

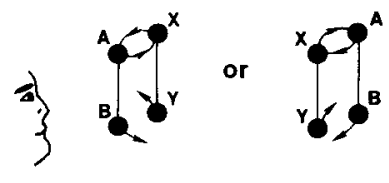

$\mathbf{B}$

Figure 2. Panels A and B show the problem of depth order in deriving interpretations for the event depicted in Figure 1. Even when the two rigid pairs of point-lights, $\mathrm{AB}$ and $\mathrm{XY}$, are seen as moving in different planes of depth, depth order is ambiguous. As shown in panel $A$, points $A B$ may be moving in front of or behind points $X Y$. Panel $B$ depicts another interpretation in which $A B$ and $X Y$ are revolving in depth, thereby continuously changing their depth order. 
tremely multistable and that the recoil percept depicted to the left in Figure 1C was often reported. Introducing reference markers that uniquely identified each element eliminated the recoil interpretation and facilitated shape discrimination for the configurations. The reference markers served to define depth relations but not depth order. That is, they did not constrain the depth order and rotational direction ambiguity depicted in Figure 2. Occlusion functions as a more powerful constraint than reference markers by defining depth order under the assumption that an occluded element is always more distant from the observer than a nonoccluded element appearing at the same spatial location (Gibson, Kaplan, Reynolds, \& Wheeler, 1969). Braunstein, Andersen, and Riefer (1982; Andersen \& Braunstein, 1983) directly examined sensitivity to dynamic occlusion as a constraint on perceived multistability. Their display consisted of elements moving as if attached to an unseen revolving sphere. Using perceived direction of rotation as their dependent measure, they found that depth order ambiguity was greatly reduced by the introduction of dynamic occlusion.

In view of these considerations, we propose an occlusion constraint as an auxiliary to the previously described model based upon a fixed axis assumption (see Figures 1 and 2). If point-lights $X Y$ are made to disappear and appear just prior to and following their coincidence with $A B$, and this appearance and disappearance is taken as representing the occlusion of point-lights $X Y$ by some structure lying between the planes containing $\mathrm{AB}$ and $\mathrm{XY}$, then a unique solution is determined because the depth order of the point-lights is specified. ${ }^{1}$ The recoiling interpretation on the left of Figure 1C is eliminated because the continuous paths of the closest point-lights are not interrupted by a collision with the more distant points. The depth order ambiguity depicted in Figure 2 is eliminated because the appearing and disappearing points are always seen as being more distant than those that are not occluded.

\section{Statement of the Problem}

As representations of three-dimensional objects, pointlight walker displays are infinitely ambiguous. Processing models that have focused solely on motion and topographical parameters have failed to specify a unique interpretation for these displays. By combining an occlusion constraint with a fixed axis assumption, a unique solution can be obtained.

Two experiments were conducted. The first represented a direct test of whether or not laterally viewed point-light walker displays without occlusion are multistable, and whether or not occlusion affects this perceived ambiguity. The second experiment was designed to address more carefully the specific effects of occlusion on point-light walker displays.

In these experiments, we elected to use a reduced form of the point-light walker display. This stimulus was a computer-generated array of 11 point-lights, moving as if attached to the head and major joints of a person walking on a treadmill. This display differed from Johansson's naturally produced displays in a number of important respects, including the former's lack of: (1) translation, (2) higher harmonic motions produced by the minor joints of the foot and ankle, and (3) natural asymmetries in posture and gait. Although we suspect that all of these natural motion parameters facilitate person recognition in point-light walker displays, we chose to eliminate them for two reasons. First and most importantly, we wanted to utilize a display that possessed all of the motion information required by all of the various processing models from the psychological and computer vision literatures; however, we did not want the display to contain any additional effective information for person identification. Our stimulus satisfied this requirement. Second, we wanted to assess whether or not observers would make use of occlusion information and, therefore, we needed to use a display that afforded some degree of multistability. Thus, although the display chosen was sufficiently compelling for all observers to see a walking person when viewing it with translation (Proffitt, 1983), translation was eliminated because none of the processing models required it and the stimulus without translation provided a greater opportunity for occlusion to affect multistability.

\section{EXPERIMENT 1: OCCLUSION VERSUS NO OCCLUSION}

This experiment addressed two questions: First, will a point-light walker display that lacks occlusion be perceived as multistable? Given an affirmative answer to this first question, the second question asks whether or not occlusion will serve to reduce this perceived ambiguity. In particular, we presented point-light walker displays, with and without occlusion, to observers and had them make judgmients on perceived multistability. We predicted that, without occlusion, the displays would be perceived as multistable and that the addition of occlusion would reduce this perceived ambiguity. We also presented scrambled versions of the two walker displays. These served as controls for nonspecific cues that might affect observers' judgments.

\section{Method}

Subjects. Forty-eight University of Virginia undergraduates participated as part of a demonstration in their experimental psychology course. They were assigned equally to four groups and tested in groups.

Stimuli. Four dynamic displays were utilized: Two were canonical point-light walker displays, one with and the other without occlusion, and the other two were scrambled versions of the canonical displays. Both the canonical and scrambled displays consisted of 11 moving pointlights. As depicted in Figure 3A, the canonical displays had the lights moving as if attached to the head and major joints of a walking person. In the case of the occlusion stimulus, the point-lights on the elbow, wrist, knee, and ankle of the body's far side turned on and off as they would if the body was present to occlude them. Also, the near hip light was periodically occluded by the near arm. The far shoulder and far hip pointlights were not present in either the occlusion display or the nonocclusion display. As Figure 3B shows, the scrambled displays preserved the absolute motions of the preceding display, but the relative locations of the lights were randomly varied. Thus, these anomalous stimuli presented motions identical to those in the canonical walker and, in the 
A

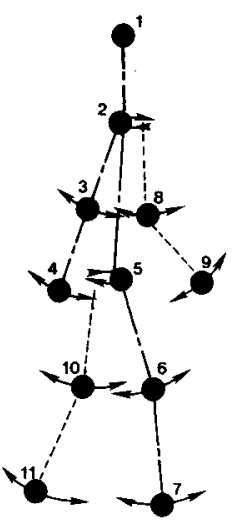

B
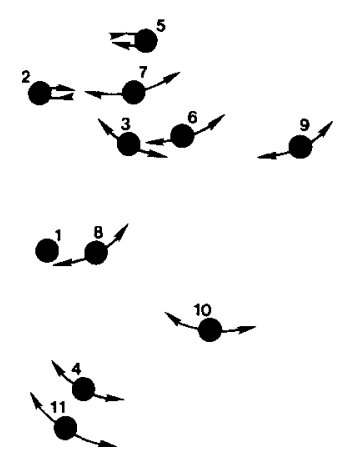

Figure 3. Depicted in panel $A$ is the canonical display of 11 pointlights moving as if attached to the head and major joints of a walking person. The motion vectors drawn through each point represents the perceived relative motions within the figure. The scrambled walker, depicted in panel $B$, is identical to $A$ except that the relative locations of the point-lights have been scrambled. Correspondingly, numbered points in the two panels have the same absolute motions.

case of the occlusion stimulus, the appropriate point-lights turned on and off twice per step cycle. Neither the fixed axis nor the occlusion constraint could be applied to the scrambled display. ${ }^{2}$ Thus, these scrambled displays insured that differential responding to occluding and nonoccluding displays was not a function of any nonspecific effect of the point-lights' blinking on and off. The displays were created through computer synthesis (Cutting, 1978) on a Hewlett-Packard (HP) 1000L computer with an HP $1350 \mathrm{~A}$ display system, videotaped, and presented to observers on a $48.3-\mathrm{cm}$ video monitor.

Design. The observers were divided into four groups; two groups saw the canonical displays and two saw the scrambled stimuli. The groups were further defined by whether they saw the occlusion or nonocclusion display first. All observers first viewed one of the displays and then made judgments about its multistability. The observers then saw the other version of the same event and made the same judgments on perceived ambiguity.

Procedure. The experiment began with a preliminary phase in which the concept of multistability was demonstrated. Three examples were shown: Rubin's (1921) "faces-vase" picture, the Necker cube, and a video display of revolving point-lights previously found to be multistable (Proffitt, 1981). The observers were then shown one of the four pointlight displays for $1.5 \mathrm{~min}$. While viewing this display, the observers were instructed to make a check mark each time their perception changed from one stable percept to another.

Following the stimulus presentation, the observers were asked to write answers to a series of questions: (1) Briefly describe what you just saw on the TV. (2) Rate on a scale from 1 to 7 how new or different the interpretations that you saw were from each other. (Observers were asked to consider a Necker cube that has only two similar interpretations as being a 1, and a cloud formation that may be seen as having an indefinite number of novel interpretations as being a 7.) (3) How many different interpretations did you see? (4) Rate on a scale from 1 to 7 how three-dimensional the stimulus appeared to you, 1 being two-dimensional and 7 being threedimensional.

Finally, the observers were given a drawing that showed 11 dots configured as if they were a static image taken from the step cycle of the stimulus they had just seen. The observers were asked to connect the dots in a manner suggested to them by the previously observed displays.

After completing these tasks, the observers were shown, for $1.5 \mathrm{~min}$, either the occlusion or the nonocclusion version of the previous display. The procedure of checking organizational changes, answering questions, and connecting dots that had been employed in the first stimulus presentation was followed during this second viewing.

\section{Results}

Multistability was assessed during the viewing sessions by having observers make checkmarks every time they perceived a change in the organization of the stimulus displays. A mixed design analysis of variance was used to test whether condition (canonical vs. scrambled), occlusion status (occlusion vs. no occlusion), or order (occlusion followed by no occlusion or vice versa) was systematically related to the number of perceived organizational changes. Condition and order represented the between-subjects variables, and occlusion status represented the repeated measure.

The principal finding from this analysis was a significant interaction between occlusion status and condition $[\mathrm{F}(1,44)=4.29, \mathrm{p}<.05$; see Figure 4]. Planned comparisons revealed that there were significantly more organizational changes to the no occlusion than to the occlusion display in the canonical condition $[F(1,44)=$ $5.1, \mathrm{p}<.03$ ], whereas there were no significant differences between the occlusion and no-occlusion displays in the scrambled condition $[\mathrm{F}(1,44)=.45$, n.s.; $\alpha=.05]$. This last finding eliminates the possibility that the difference in the number of perceived organizational changes between occlusion and no-occlusion displays could be simply attributed to the blinking of some of the lights in the occlusion displays. The number of perceived organizational changes in the canonical versus the scrambled condition was not significantly different $[F(1,44)=2.83$, n.s.].

Following each viewing session, the observers were asked to describe what they had seen. The most dramatic result from this query was the number of observers who identified a walking person after viewing one of the canon-

\section{EXPERIMENT 1}

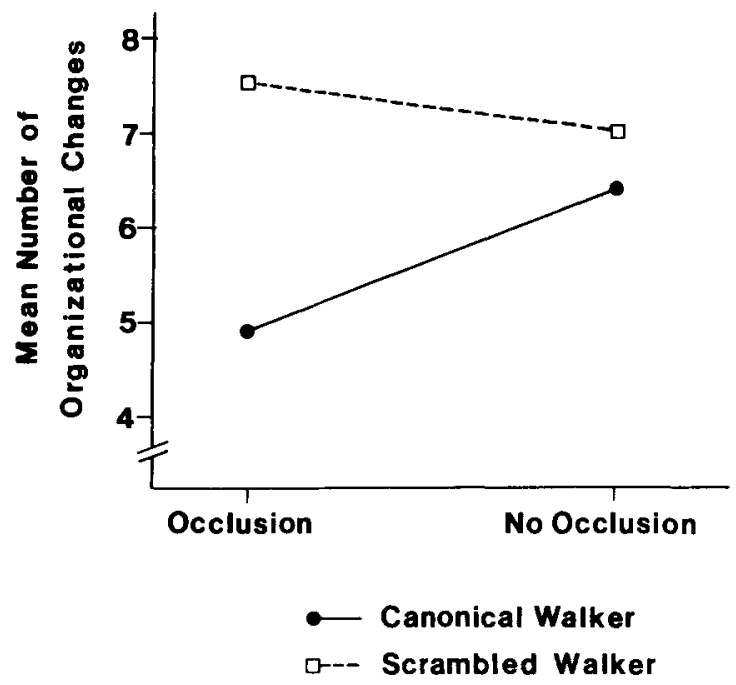

Figure 4. The results in Experiment 1 are shown for the task assessing multistability in which observers recorded the number of times they perceived a change in the organization of the stimulus display. 
ical displays for the first time: $83 \%$ of the observers shown the occlusion display recognized it as a person walking, whereas only $33 \%$ did so after viewing the no-occlusion stimulus $(\mathrm{Z}=2.5, \mathrm{p}<.02)$. Results from the second viewing session showed a strong carryover effect; when the no-occlusion display was shown second, walker recognition increased by $34 \%$. When not seen as a walker, both of these displays were described in a variety of ways, including lights moving as if attached to strings, oscillating back and forth, or rotating. Interestingly, $25 \%$ of the observers shown the no-occlusion display described what they saw as balls bouncing off of each other. As previously discussed, this description was frequently given to similar displays in which relative depth information was absent (White \& Mueser, 1960).

Two other measures of multistability were analyzed using the same statistical design as used with the checkmark data. These measures of the newness and the number of interpretations proved to be less sensitive than the checkmark measure. Although scrambled displays were seen as having both a greater number of and more novel interpretations than the canonical walkers $[\mathrm{F}(1,44)=6.01$, $p<.02$, and $F(1,44)=4.50, p<.04$, for number and newness judgments, respectively], the principal comparison between the occlusion and no-occlusion canonical display was nonsignificant. The newness ratings did show the predicted trend, with the occlusion displays having a mean rating of 2.5 and the no-occlusion walkers having a mean of 3.58 .

The final question required observers to rate the threedimensionality of the displays on a 7-point scale ( 1 being 2-D and 7 being 3-D). A comparison between the two canonical displays revealed no difference $[F(1,44)=$ 2.79 , n.s.]. The means for the occlusion and no-occlusion displays were 5.2 and 4.7 , respectively. Similarly, no differences were found for the scrambled stimuli $[F(1,44)$ $=.11$, n.s.]. The means for the occlusion and noocclusion displays were 4.5 and 4.6 , respectively.

The last measure was based upon the connect-the-dots task. Each drawing in the canonical condition was scored for the total number of lines added or deleted relative to the correct pattern of 10 lines connecting the major joints and head of a person. A comparison of these scores as a function of occlusion status revealed no significant effects; however, the trend was in the expected direction. Mean additions and deletions for occlusion and noocclusion displays were 3.0 and 3.9 , respectively.

\section{Discussion}

These results provide affirmative answers to the two questions that motivated the experiment: Point-light walker displays lacking occlusion were perceived as multistable, and the addition of occlusion served to reduce this perceived ambiguity. Occlusion information further served to facilitate the recognition of a walking person in the canonical displays. These results indicate that processing models for identifying point-light walker displays must include additional constraints beyond those that have presently been proposed. In particular, observers utilize occlusion when it is present, and thus, rules for processing this information could reasonably be added to such existing processing constraints as the fixed axis assumption.

The finding that occlusion did not affect judgments of three-dimensionality is consistent with the findings of Andersen and Braunstein (1983). In their studies of the kinetic depth effect, they found that occlusion reduced perceived ambiguity of rotational direction and depth order, but did not affect perceived relative depth. Our results extend the generality of their findings.

Even when occlusion was present in the canonical display, our results show the presence of multistability, and even after a 1.5-min viewing session, some observers still did not recognize that the stimulus could be seen as depicting a walking person. This latter finding was, to us, startling in light of Johansson's (1976) demonstration that 0.1-0.2 sec was a sufficient exposure duration for perceiving the human form in point-light displays. Recall that Johansson created his stimuli by attaching point-light sources to real walkers. This lack of concordance with the earlier research suggests that, like occlusion, there must have been additional information present in Johansson's displays that observers utilized in forming their perceptions. Likely candidates for such information found in natural, but not in our computer-generated displays are: translation, higher frequency harmonic motions produced by the minor joints of the body, asymmetries in the body's posture, and asymmetries in the gait from one step to another. Additional research is required to examine the role played by these and other informational sources in specifying the unique percept of a locomoting person in point-light walker displays. We know that translation, for example, facilitates walker recognition (Proffitt, 1983); however, none of the existing processing models utilize translation, and, unlike occlusion, we cannot at present propose a general interpretative constraint that can derive connectivity from translation information.

\section{EXPERIMENT 2: APPROPRIATE VERSUS INAPPROPRIATE OCCLUSION}

The previous experiment demonstrated that occlusion can play a role in reducing ambiguity and facilitating identification in point-light walker displays. We sought next to define more precisely the function that this information served in organizing observers' perceptions. Two possibilities could not be resolved from the findings of Experiment 1. One possibility is that, although both canonical displays were seen as equally three-dimensional, the specification of depth order by occlusion delimited the number of perceived interpretations. For example, $360^{\circ}$ rotations, as depicted in Figure 2B, are much more difficult to see in the occluding display. The other possibility is that occlusion information not only specifies the depth order of different point-lights, but also specifies por- 
tions of the external contour of the implicit occluding form. To examine these two possibilities, we replicated the design of the first experiment with the modification that, instead of using the no-occlusion stimuli, we employed displays in which occlusion occurred in a manner inconsistent with any obvious underlying structure such as that of the human form. Should these appropriate and inappropriate occlusion stimuli prove to be equally stable, then we would infer that the role of occlusion was primarily one of specifying depth order. Should the inappropriate occlusion display be found to be more multistable, then we would conclude that occlusion served also as a source of information about the implicit occluding form of the walker's body.

\section{Method}

Subjects. A total of 56 University of Virginia undergraduates participated as part of a class demonstration in their experimental psychology course. They were assigned equally to one of four groups and tested in groups.

Stimuli. Four computer-generated displays of point-lights were used. The first two were used in Experiment 1 and corresponded to the canonical and scrambled displays with occlusion. The latter two displays consisted of point-lights programmed to appear and disappear at times during the step cycle that were inappropriate for the structure of the human form. Figure 5A shows the path and occlusion period for the point-lights on the far side of an appropriate occlusion walker, and Figure $5 \mathrm{~B}$ does likewise for the inappropriate display. The appropriate and inappropriate occlusion displays were identical with respect to which point-lights blinked on and off and the duration of disappearance; they differed only with respect to the portion of the step cycle during which the blinking occurred. One inappropriate occlusion display was created using the canonical walker, and the other was created using the scrambled stimulus. The inclusion of the two scrambled displays provided a control for the possibility that differences in judged multistability could be a function of phase differences in the blinking of point-lights.

Design and Procedure. The design and procedure was identical to those of Experiment 1, except for the change in stimuli. Two groups were shown the two canonical displays, and two were shown the two
A

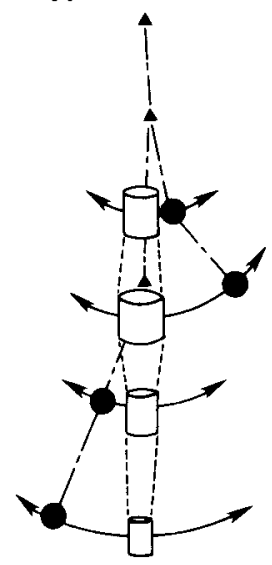

B

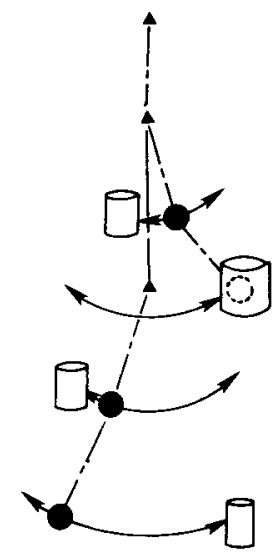

Figure 5. The cylinders in panels $A$ and $B$ show the locations where the point-lights disappear for the appropriate and inappropriate occlusion displays, respectively. The four point-lights on the far side of the body are shown moving behind these cylinders, which occlude them from view.
EXPERIMENT 2

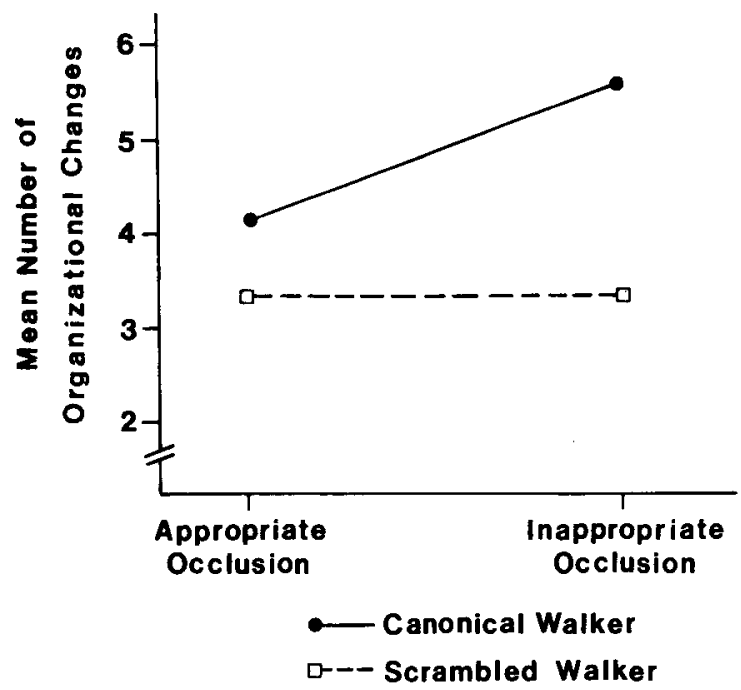

Figure 6. The results in Experiment 2 are shown for the task assessing multistability in which observers recorded the number of times they perceived a change in the organization of the stimulus display.

scrambled stimuli. The groups were further defined by the presentation order of the two displays.

\section{Results}

Multistability was again assessed using a mixed design analysis of variance with number of checkmarks as the dependent measure. Condition and order served as the between-subjects variables, and occlusion status (appropriate or inappropriate occlusion) represented the repeated measure. A main effect was revealed for occlusion status, with appropriate occlusion displays producing significantly fewer organizational changes than the inappropriate occlusion displays $[\mathrm{F}(1,52)=4.27$, $\mathrm{p}<.05]$. Differential responding as a function of occlusion was not equal for both conditions. As can be seen in Figure 6, observers perceived fewer organizational changes in the appropriate occlusion displays than in the inappropriate ones for the canonical condition $[F(1,52)$ $=7.72, \mathrm{p}<.01]$, but showed no difference in their responses to scrambled displays $[F(1,52)=0.0$, n.s.]. The number of perceived organizational changes in the canonical versus the scrambled condition was not significantly different $[F(1,52)=3.17$, n.s. $]$.

The difference between the two canonical displays received additional support from the data collected from the task requesting that observers describe what they had seen. When the first display shown was the walker display with appropriate occlusion, $87 \%$ of the observers described it as a person walking; on the other hand, when the first display shown was the canonical display with inappropriate occlusion, only $50 \%$ of the observers recognized it as a walker. This difference in recognition of the 
two point-light displays was significant $(Z=2.21$, $\mathrm{p}<$.04). Results from the second viewing again showed a carryover effect; when the inappropriate occlusion display was shown second, walker recognition increased to $68 \%$.

The other measures-newness and number of interpretations, and connection of the dots-revealed no significant effects. Also, there were no significant differences in rating the three-dimensionality of the displays. For the canonical condition, the mean 3-D ratings for the appropriate and inappropriate occlusion displays were 5.13 and 4.98, respectively; for the scrambled condition, the mean 3-D ratings for the appropriate and inappropriate occlusion displays were 3.81 and 3.86 .

\section{Discussion}

Once again, significant differences were observed in response to the two canonical displays, whereas no differences were found between the two scrambled displays. For those observers who saw the canonical point-light walker displays, appropriate occlusion served to reduce multistability and increase the likelihood of recognizing the display as a walking person. These findings are particularly impressive when one considers that the only difference between the two canonical displays involved the spatial-temporal patterning of blinking among five of the point-lights. As such, the two displays differed neither in the amount of blinking nor in the specification of depth order as a function of perceiving the blinking lights in terms of occlusion. Rather, these two displays differed only with regard to the implicit occluding forms that were defined. It is therefore concluded that occlusion is used not only to specify depth order, but also to define implicit occluding contours.

Reduced ambiguity in the appropriate occlusion walker was probably due, in large part, to the perceptual stability of the walking-person interpretation that is typically seen in this display. Our own experience suggests that once such an interpretation is achieved, it is less likely that other interpretations will be perceived. The greater frequency of person recognition among those who viewed the appropriate occlusion displays may have been due to either specific or more general factors. One possibility is that identification was directly facilitated by the congruence of occlusion with the structure of the human body-the point-lights in the appropriate occlusion display blink on and off in the familar pattern associated with a walking person. The other possibility is that identification was indirectly facilitated by the good continuation of occluding contours present in the appropriate, but not in the inappropriate, display. An examination of Figure 5 shows that the occluding contours line up in a simple manner, depicted by dotted lines, only in the appropriate occlusion display. This good continuation may have served to specify a single implicit form that could easily be interpreted as the human body.

The lack of differences in judged three-dimensionality again suggests that occlusion is not a necessary condition for perceiving relative depth. Rather, occlusion functions to specify depth order and the presence of implicit occluding forms.

\section{CONCLUSION}

Our understanding of the role played by motion information in organizing the visual world has been greatly facilitated by the use of such reduced stimuli as moving point-light displays. By reducing to a minimum all optical information except motion, researchers have demonstrated that observers can utilize motion in perceiving many environmental properties. Still, we have much to learn about how motion specifies various properties of the environment. Reduced stimuli are inherently ambiguous when considered from a formal mathematical perspective; current research suggests that stable perceptions of these displays must be based not only upon proximal stimulation, but also upon the interpretative constraints manifest in active perceptual processing.

Our experiments examined the effect on multistability of the blinking on-and-off of a subset of lights in pointlight walker displays. We found that observers took the appearance and disappearance of these lights as representing occlusion, which was used to specify both depth order relations and implicit occluding forms. All existing processing models for extracting connectivity from pointlight displays of moving jointed objects rely solely on motion and topographical information present in our nonoccluding walker display; in so doing, these models seek to derive a unique perception from information that is seen by observers as being multistable. Our research emphasizes the need to modify these models to include sensitivity to additional interpretative constraints.

It needs to be further noted that, even in the occlusion conditions, our point-light walker display was not always recognized as a locomoting person, and multistability was still present. It seems likely to us that the number of occluded point-lights in our display was near the minimum required to create the impression of occlusion, and that the stimulus event could be altered in such a manner as to increase occlusion salience. Increasing the size of the point-lights, for example, might increase the impression of occlusion, which, in turn, could reduce multistability. Supporting this supposition are the findings of Andersen and Braunstein (1983) that perceived multistability is lessened by conditions that increase the salience of occlusion.

Recognizing a person after only a brief exposure to a point-light walker display is a remarkable achievement for the human perceptual system. Surely, a successful modeling of the perceptual processes that perform this feat will be informative about more general issues in perceptual functioning. However, before psychological processing models can successfully be advocated, we need to know more about the necessary stimulus condition for identifying the human form in these displays. Our research indicates that we do not currently know what the minimal conditions are for perceiving a unique impression of a 
locomoting human in point-light stimuli. Most likely, these minimal conditions will vary with the number and combination of constraints placed on the distal event. Thus, increasing the salience of occlusion may result in the elimination of multistability, or alternatively, the present amount of occlusion may be sufficient if other stimulus information is included that gives these displays more of the appearance of Johansson's $(1973,1976)$ naturally produced point-light walker displays.

\section{REFERENCES}

AgGarWal, J. K., \& Martin, W. N. (1981). Analyzing dynamic scenes containing multiple moving objects. In T. S. Huang (Ed.), Image sequence analysis. Berlin: Springer.

ANDersen, G. J., \& Braunstein, M. L. (1983). Dynamic occlusion in the perception of rotation in depth. Perception \& Psychophysics, 34, 356-362.

AtTNEAVE, F. (1971). Multistability in perception. Scientific American, 225, 62-71.

Bertenthal, B. I., \& Kramer, S. (1984). The TMS 9918A VDP: A new device for generating moving displays on a micorocomputer. Behavior Research Methods, Instruments, \& Computers, in press.

Braunstein, M. L. (1983). Perception of rotation in depth: The psychophysical evidence. In Motion: Representation and perception (ACM SIGGRAPH/SIGART Interdisciplinary Workshop). New York: Association for Computing Machinery.

Braunstein, M. L., Andersen, G. J., \& Riefer, D. M. (1982). The use of occlusion to resolve ambiguity in parallel projections. Perception \& Psychophysics, 31, 261-267.

Cutring, J. E. (1978). A program to generate synthetic walkers as dynamic point-light displays. Behavior Research Methods \& Instrumentation, 10, 91-94.

CUTTING, J. E., \& KozLowski, L. T. (1977). Recognizing friends by their walk: Gait perception without familiarity cues. Bulletin of the Psychonomic Society, 9, 353-356.

Cutting, J. E., \& ProffitT, D. R. (1981). Gait perception as an example of how we may perceive events. In R. Walk \& H. L. Pick, Jr. (Eds.), Intersensory perception and sensory integration. New York: Plenum.

Cutting, J. E., \& Proffitt, D. R. (1982). The minimum principal and the perception of absolute, common, and relative motions. Cognitive Psychology, 14, 211-246.

Cutting, J. E., Proffit, D. R., \& Kozlowski, L. T. (1978). A biomechanical invariant for gait perception. Journal of Experimental Psychology: Human Perception and Performance, 4, 357-372.

Gibson, J. J., Kaplan, G. A., ReYNolds, H. N., \& WheELeR, K. (1969). The change from visible to invisible: A study of optical transition. Perception \& Psychophysics, 5, 113-116.
Hoffman, D. D., \& Flinchbaugh, B. E. (1982). The interpretation of biological motion. Biological Cybernetics, 42, 195-204.

Johansson, G. (1973). Visual perception of biological motion and a model for its analysis. Perception \& Psychophysics, 14, 201-211.

JoHANSSON, G. (1976). Spatio-temporal differentiation and integration in visual motion perception. Psychological Research, 38, 379-396.

Metzger, W. (1934). Tiefenerscheinungen in optischen Bewegungsfeldern. Psychogische Forschung, 20, 195-260.

O'ROURKE, J., \& BADLER, N. I. (1980). Model-based image analysis of human motion using constraint propagation. IEEE Transactions on Pattern Analysis and Machine Intelligence, PAM1-2, 524-536.

ProfFit, D. R. (1981). Kinetic and proximity influences on perceiving structure in revolving point-lights. Paper presented at 22nd Annual Meeting of the Psychonomic Society, Philadelphia.

ProfFIT, D. R. (1983). Factors affecting multistability in moving pointlight displays. Paper presented at 24th Annual Meeting of the Psychonomic Society, San Diego.

ProffitT, D. R., \& CutTing, J. E. (1980). An invariant for wheelgenerated motions and the logic of its determination. Perception, 9 , 435-449.

Restle, F. (1979). Coding theory of the perception of motion configurations. Psychological Review, 86, 1-24.

RuBIN, E. (1921). Visuell wahregenomenne Figuren. Copenhagen: Gyldendalske.

UlLMAN S. (1979). The interpretation of visual motion. Cambridge, MA: M.I.T. Press.

Wallach, H., \& O'ConNell, D. N. (1953). The kinetic depth effect. Journal of Experimental Psychology, 45, 205-217. (Also in H. Wallach, On perception. New York: Quadrangle, 1976)

WEBB, J. A., \& AGGARWAL, J. K. (1982). Structure from motion of rigid and jointed objects. Artificial Intelligence, 19, 107-130.

White, B. J., \& Mueser, G. E. (1960). Accuracy in reconstructing the arrangement of elements generating kinetic depth displays. Joumal of Experimental Psychology, 60, 1-11.

\section{NƠTES}

1. This interpretation does not include a specification of absolute size or relative depth.

2. The fixed axis assumption cannot be applied, since the radius of rotation for one point about another (an elbow about a shoulder, for example) is never equal to the distance between the two points. The occlusion constraint cannot be applied, since the path through which a point passes during the interval in which it is "off" is never intersected by another point that is "on."

(Manuscript received March 26, 1984; revision accepted for publication July 24, 1984.) 